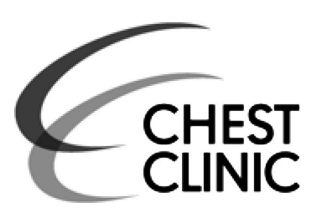

AUDIT, RESEARCH AND GUIDELINE UPDATE

\title{
Obtaining real-world evidence: the Salford Lung Study
}

\author{
John P New, ${ }^{1}$ Nawar Diar Bakerly, ${ }^{1}$ David Leather, ${ }^{2}$ Ashley Woodcock ${ }^{3}$
}

${ }^{1}$ Salford Royal NHS Foundation

Trust, Salford, UK

${ }^{2}$ GlaxoSmithKline UK Ltd, Stockley Park West, Uxbridge, Middlesex, UK

${ }^{3}$ Institute of Inflammation and Repair, Manchester Academic Health Science Centre, University of Manchester, Manchester, UK

\section{Correspondence to} Professor Ashley Woodcock, Institute of Inflammation and Repair, 2nd Floor Education and Research Centre, University Hospital of South Manchester NHS Foundation Trust,

Southmoor Road, Manchester M23 9LT, UK; ashley. woodcock@manchester.ac.uk

Received 7 February 2014 Accepted 10 February 2014 Published Online First 6 March 2014

\section{( OPEN ACCESS}

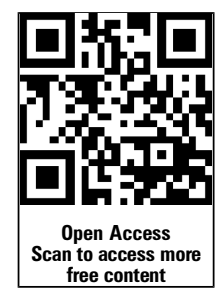

CrossMark

To cite: New JP

Bakerly ND, Leather $D$, et al.

Thorax 2014;69:

$1152-1154$.

\section{ABSTRACT}

We need to assess clinical treatments in real-life settings outside of randomised controlled trials (RCTs). Pragmatic RCT (pRCT) data can supplement RCTs by providing effectiveness information to support healthcare decisions. Electronic health records can facilitate concurrent safety monitoring and data collection without direct patient contact for large randomised study populations in PRCTs. The Salford Lung Study is the world's first phase III PRCT in asthma and chronic obstructive pulmonary disease (COPD), which aims to randomise over 7000 patients. This paper describes the hurdles overcome and the enormous effort and resource required to establish this comparative effectiveness study of a prelicence intervention.

GlaxoSmithKline protocol HZC115151 Asthma study clinicaltrials.gov registration NCT01706198

COPD study clinicaltrials.gov registration NCT01551758

\section{INTRODUCTION}

Double-blind randomised controlled trials (RCTs) are considered the most robust form of medical evidence, and their data form the basis of regulatory approval of new therapies and their incorporation in clinical guidelines. However, additional information is needed about the risk/benefit profile of new treatments in real-world practice, and especially evidence providing relative 'values' on novel medications, in cash-limited health services.

We designed the Salford Lung Study, the world's first prelicence pragmatic RCT ( $\mathrm{pRCT}$ ) to compare the real-world effectiveness in routine primary care of a novel once-daily investigational treatment (vilanterol/fluticasone furoate in a new dry-powder inhaler (DPI), Ellipta) with existing therapy for chronic obstructive pulmonary disease (COPD) and asthma. Here we discuss how this pRCT differs from traditional RCTs and retrospective database analyses.

\section{THE NEED FOR BETTER EVIDENCE}

A standard RCT aims to establish efficacy-an unequivocal cause-and-effect relationship for a very limited number of predefined outcomes. The interventions are double blind, often with double dummy to maintain blinding, in an idealised setting. Well-defined and compliant patients with minimal comorbidities are closely supervised for $>90 \%$ adherence and to minimise confounding factors that could compromise the primary outcome, on which a regulatory approval may critically depend. However, the proportion of patients with asthma and COPD who meet the common inclusion criteria in RCTs has been estimated to be as low as $3 \%$ and $7 \%$, respectively. ${ }^{1}$ It is extremely difficult to extrapolate data from an RCT into real life for a number of reasons, including patient preference, lower adherence and comorbidities.

In contrast, 'real-world' studies assess effectiveness in large unselected populations, which include patients with comorbidities. Patients are under routine care, taking open-label treatment over a prolonged period, with no additional visits and no attempt to change adherence. Data are usually obtained using electronic health records, which provide long-term outcomes, including health economics, free from interviewer or recall bias. However, most effectiveness research is retrospective, limited by its non-randomised nature, and more robust study designs are required. ${ }^{2}$ The International Society for Pharmacoeconomics and Outcomes Research has made recommendations on prospective observational studies for clinical effectiveness research. ${ }^{3}$ Its report defines different types of clinical effectiveness research studies, including retrospective and prospective observational studies, and pRCTs.

pRCTs are large prospective clinical studies in which patients are randomised to two or more interventions, and then followed up according to the investigating physicians' usual practice. ${ }^{4}$ pRCTs bridge the gap between RCTs and non-randomised observational studies, allowing a good approximation of real-world practice. An electronic health record can be used to identify patients and assess outcomes, and provide safety surveillance. However, pRCTs require enrolment of a broad population to maximise external validity and can be expensive and difficult to implement. One important limitation is the likelihood of treatment switching during the trial, which can reduce the value of randomisation.

The past two decades have seen an increased number of published reports of pRCTs (43 in 1990 to 252 in 2010) across a range of therapy areas, although they still represent $<1 \%$ of all randomised trial reports. ${ }^{4}$ The Salford Lung Study is the first attempt to carry out a pRCT prior to registration of a new treatment.

\section{THE SALFORD LUNG STUDY}

The Salford Lung Study is an open-label phase III $p R C T$ of a once-daily long-acting $\beta$ agonist (LABA)/inhaled corticosteroid DPI, in asthma and COPD. Robust effectiveness evidence would be of particular value in assessing once-daily inhaled therapies for asthma and COPD, where improved 
outcomes may result from better adherence. Patients are randomised to receive either a continuation of their usual treatment or a novel once-daily DPI containing a combination of a new inhaled steroid and a new LABA (fluticasone furoate/vilanterol (FF/VI; GlaxoSmithKline, Stockley Park, UK)) for 12 months. The primary outcome for COPD is the rate of moderate and/or severe exacerbations, and in the asthma study, an improvement in asthma control (Asthma Control Test). A full regulatory package for FF/VI is under consideration by the European Medicines Agency, and at the time of study initiation extensive efficacy and safety data were already available for more than 6400 patients from completed RCTs.

After randomisation, patients receive 'usual' care for 12 months by their own general practitioner (GP), practice nurse and community pharmacist. Effectiveness and safety data are monitored and collected in near-real time using an electronic health record, minimising the number of patient visits required. GPs prescribe as usual, patients order and collect repeat prescriptions in their usual way, and collect their study medication from their usual community pharmacist.

Salford is a metropolitan borough of Greater Manchester, UK. A number of clinical and infrastructure factors contribute to making it a unique environment for conducting a pRCT of a new therapeutic agent in respiratory medicine in a pre-licensing timeframe. Salford has a relatively static population served by a single hospital (Salford Royal Hospital). An integrated electronic health record connects the hospital and surrounding primary care practices in real time. The Salford Integrated Record (SIR), established in 2001, captures linked data in real time on all people accessing primary and secondary care services in Salford. The NorthWest e-Health group (NWeH; see http://www.nweh. org.uk/), which comprises information technology specialists, clinicians, researchers, epidemiologists and project managers, has developed a methodology and governance framework using data from the SIR in effectiveness research. In a pilot study, we successfully quantified the burden of asthma and COPD, identified exacerbations and evaluated differences in disease outcomes and healthcare resource use in Salford. ${ }^{5}$ For the Salford Lung Study, additional data feeds have been added to capture access of out-of-hours services, access to health services outside of Salford and deaths. To ensure accuracy, all of the possible adverse events or study endpoints are verified blind by the study clinicians. Thus, we can accurately evaluate composite endpoints of primary and secondary care events while closely monitoring patient safety. Uniquely, the SIR links primary and secondary care with patient-level prescription information. All 55 of Salford's community pharmacies are participating in the Salford Lung Study, which allows assessment of real-world medication adherence in terms of number of prescriptions delivered to the pharmacy and the number dispensed to the patient. Salford benefits from a unified primary and secondary care COPD pathway, a common template for COPD assessment and a communitybased respiratory physician working across primary and secondary care units, within an area of high need.

\section{STUDY DESIGN CHALLENGES}

The key objectives in designing and executing the Salford Lung Study are to enable collection of data with minimal disruption to normal care, enrol a large proportion of the local patient population, provide appropriate safety monitoring and meet all ethical and regulatory requirements. In the study planning stages, the study sponsor and partners took advice from independent experts, including the Medicines and Healthcare Products Regulatory Agency (MHRA), the National Institute for Health and Care Excellence (NICE) and the National Institute for Health Research (NIHR), as well as the National Research Ethics Service Committee North West, Greater Manchester South.

Phase III trials necessitate stringent safety monitoring. We are able to provide this in the context of usual care by remote monitoring using the SIR. Formal study visits are only required at baseline (consent and randomisation) and at study end. Telephone calls every 3 months act as a 'safety net' if there has been no other contact between a patient and their GP.

We have had to overcome major challenges. In order to enrol a large proportion of the population, we found that GPs and practice nurses had to become responsible for recruitment of their own patients. The amount of patient information required for phase III informed consent is a challenge to recruitment in a study like Salford Lung Study, and the concept of taking an experimental medicine may make eligible patients reluctant to participate. The majority of participating healthcare professionals had little experience of prelicence clinical research, and a change to a research culture has taken time. Extensive training in good clinical practice was delivered to more than 1000 nurses, pharmacists and GPs from 51 general practices in Salford. A public education campaign took place in parallel. Approximately 150 GlaxoSmithKline -funded healthcare workers are engaged in the study.

The first patients were enrolled into the COPD study in April 2012, and the asthma study in December 2012. By mid-October 2013, more than 2000 patients were consented and 1600 patients were randomised in the COPD study, on schedule for a target of 2800 by end March 2014, with first results due end second quarter 2015. The asthma study recruitment is now accelerating, and likely to complete 6-12 months later. The study execution has required intensive collaboration across the National Health Service, with the NIHR, MHRA, ethics committee, academia and industry. The creation of an effectiveness study environment in Salford serves as a benchmark for other initiatives, including pharmacovigilance and phase IV studies, to collate data from primary and secondary care anywhere in the UK. We anticipate that initiatives such as this will reshape the future of clinical trials and meet the demand for value-based medical evidence.

Correction notice This article has been corrected since it was published Online First. The Medicines and Healthcare Regulatory Authority (MHRA) has been amended to read Medicines and Healthcare Products Regulatory Agency (MHRA).

Acknowledgements The authors thank Tom Westgate of iMed Comms, Macclesfield, UK, who provided medical writing support, which was funded by GlaxoSmithKline.

Contributors All authors were involved in the design and implementation of the Salford Lung Study and contributed equally to the preparation of this paper, including development of the outline, review of all drafts, final approval and decision to submit the manuscript to Thorax. Information for this paper was based on the authors' personal knowledge and relevant published journal articles.

Funding This study is funded by GlaxoSmithKline. GlaxoSmithKline reviewed the manuscript for factual accuracy and funded medical writing services.

Competing interests JPN has received consulting fees from GlaxoSmithKline. NDB's employing organisation provides IT support to GlaxoSmithKline. He has received educational grants and speaker's fees from GlaxoSmithKline and Novartis, and support for attending educational conferences from Boehringer Ingelheim, GlaxoSmithKline and Novartis. DL is an employee of, and holds shares/stock options in, GlaxoSmithKline. AW has acted on advisory boards and provided consultancy for Almirall, Chiesi, Cytos and GlaxoSmithKline. He has received travel support to speak at an international meeting from GlaxoSmithKline. He is an investigator on cough and asthma studies for Afferent and GlaxoSmithKline.

Patient consent Obtained.

Ethics approval South Manchester.

Provenance and peer review Not commissioned; internally peer reviewed. 


\section{Chest clinic}

Open Access This is an Open Access article distributed in accordance with the Creative Commons Attribution Non Commercial (CC BY-NC 3.0) license, which permits others to distribute, remix, adapt, build upon this work non-commercially, and license their derivative works on different terms, provided the original work is properly cited and the use is non-commercial. See: http://creativecommons.org/ licenses/by-nc/3.0/

\section{REFERENCES}

1 Herland $\mathrm{K}$, Akselsen JP, Skjonsberg $\mathrm{OH}$, et al. How representative are clinical study patients with asthma or COPD for a larger 'real life' population of patients with obstructive lung disease? Respir Med 2005;99:11-19.
2 Helfand M, Berg A, Flum D, et al. for the Patient-Centered Outcomes Research Institute Methodology Committee. Draft methodology report: 'Our questions, our decisions: standards for patient-centered outcomes research'. 2012. http://pcori.org/ assets/MethodologyReport-Comment.pdf (accessed 1 Feb 2013).

3 Berger ML, Dreyer N, Anderson $\mathrm{F}$, et al. Prospective observational studies to assess comparative effectiveness: the ISPOR good research practices task force report. Value Health 2012;15:217-30.

4 Chalkidou $K$, Tunis $S$, Whicher $D$, et al. The role for pragmatic randomized controlled trials (pRCTs) in comparative effectiveness research. Clin Trials 2012;9:436-46.

5 New JP, Delderfield MR, Stein ND, et al. Assessing the burden of asthma and COPD in Salford UK: retrospective analysis using a whole population electronic medical record. Eur Respir J 2011;38(Suppl 55):732S. 


\section{Correction}

New JP, Bakerly ND, Leather D, Woodcock A. Obtaining real-world evidence: the Salford Lung Study. Thorax 2014;69:1152-4.

The following text on page 1153,

To ensure accuracy, all of the possible adverse events or study endpoints are verified blind by the study clinicians. Thus, we can accurately evaluate composite endpoints of primary and secondary care events while closely monitoring patient safety.

has been revised by authors. The correct text is as follows:

All possible serious adverse events are reviewed and verified by the study safety physicians and the investigators. Thus, we can accurately evaluate composite endpoints of primary and secondary care events while closely monitoring patient safety. The statistical reporting team and others involved in the analysis plan are blinded to the patients' individual therapies. Only those directly involved in clinical study conduct are not blinded to individual patient therapies. An independent statistical house not involved in the conduct of the study performed all safety analyses for the Independent Data Monitoring Committee. No analysis by randomised treatment is performed by the sponsor until after the analysis plan is finalised and the database has been locked.

Thorax 2015;70:1008. doi:10.1136/thoraxjnl-2014-205259corr1

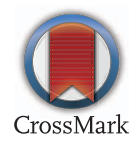

